\title{
Single Higgs production at LHC as a probe for an anomalous Higgs self coupling
}

\section{Pier Paolo Giardino*}

Brookhaven National Laboratory

E-mail: pgiardino@bnl.gov

We explore the possibility of probing the trilinear Higgs self coupling indirectly, through single Higgs production and decay processes. The method relies on the effects that electroweak loops featuring an anomalous trilinear coupling would imprint on single Higgs production at the LHC. We find that the bounds on the self coupling are already competitive with those from Higgs pair production and will be further improved in the current and next LHC runs.

38th International Conference on High Energy Physics 3-10 August 2016

Chicago, USA

${ }^{*}$ Speaker. 


\section{Introduction}

This proceeding is mostly based on [1]. The interested reader can find there detailed information on the calculations and results presented here.

The discovery of a new scalar resonance with mass around $125 \mathrm{GeV}$ at the Large Hadron Collider (LHC) opened a new era in high-energy particle physics. The study of the properties of this particle provides strong evidence that it is the Higgs boson of the Standard Model (SM).

ATLAS and CMS performed independent [2,3] and combined [4] studies on the Higgs couplings in the so-called $\kappa$-framework [5, 6]. In the combined analysis [4] the couplings with the vector bosons have been found to be compatible with those expected from the SM within a $\sim 10 \%$ uncertainty, while in the case of the heaviest SM fermions the uncertainty is of order $\sim 15-20 \%$.

The study of the trilinear $\left(\lambda_{3}\right)$ Higgs self couplings is in a completely different situation. Information on $\lambda_{3}$ can be directly obtained at LO only from final states featuring at least two Higgs bosons. However, the cross sections of these processes are much smaller than those of single Higgs production: at $\sqrt{s}=13 \mathrm{TeV}$ the single Higgs gluon-gluon-fusion production cross section is around $50 \mathrm{pb}$ [7], while the double Higgs cross section is around $35 \mathrm{fb}$ in the gluon-gluon-fusion channel [8].

In [1] we explored the possibility of constraining the trilinear Higgs self coupling via precise measurements of processes featuring single Higgs production and decay at the LHC. Indeed single Higgs production depends on $\lambda_{3}$ via weak loops, namely at Next-to-Leading (NLO) in the electroweak (EW) interactions. We therefore extract the $\lambda_{3}$-dependent part from the NLO EW corrections to all phenomenologically relevant single Higgs production cross sections and branching ratios. We obtain a distinctive pattern of deformations of the SM predictions for the rates $(\sigma(i) \cdot \mathrm{BR}(f))$, which can be compared to the experimental data.

We investigate the reach of our approach in the determination of $\lambda_{3}$ by considering the $8 \mathrm{TeV}$ Higgs data [4] and the expected performances of the forthcoming runs of the LHC $[9,10]$. We demonstrate the potential of single Higgs production channels in setting bounds on $\lambda_{3}$ that are competitive and complementary to those achievable via the searches for double Higgs production.

\section{2. $\lambda_{3}$-dependent contributions in single Higgs processes}

As basic assumption, we consider a BSM scenario where the only (or dominant) modification of the SM Lagrangian at low energy appears in the scalar potential; we concentrate in particular on the trilinear self-coupling of the Higgs boson. We therefore simply parametrise the effect of NP at the weak scale via a single parameter $\kappa_{\lambda}$, i.e., the rescaling of the SM trilinear coupling, $\lambda_{3}^{\mathrm{SM}}$ :

$$
V_{H^{3}}=\lambda_{3} v H^{3} \equiv \kappa_{\lambda} \lambda_{3}^{\mathrm{SM}} v H^{3}, \quad \lambda_{3}^{\mathrm{SM}}=\frac{G_{\mu}}{\sqrt{2}} m_{H}^{2} .
$$

The "deformation" of the Higgs trilinear coupling induces modifications of the Higgs couplings to fermions and to vector bosons at one loop. Since such loop-induced $\lambda_{3}$-dependent contributions are energy- and observable-dependent, the resulting modifications cannot be parameterised via a rescaling of the tree-level couplings of the single Higgs production and decay processes considered. Thus, the effects cannot be correctly captured by the standard $\kappa$-framework $[5,6]$. 


\begin{tabular}{|c|c|c|c|c|c|c|c|c|c|c|c|}
\hline$C_{1}^{\sigma}[\%]$ & $g g \mathrm{~F}$ & VBF & $W H$ & $Z H$ & $t \bar{t} H$ & & & & & & \\
\hline $7 \mathrm{TeV}$ & 0.66 & 0.65 & 1.06 & 1.23 & 3.87 & $C_{1}^{\Gamma}[\%]$ & $\gamma \gamma$ & $Z Z$ & $W W$ & $f \bar{f}$ & $g g$ \\
\hline $8 \mathrm{TeV}$ & 0.66 & 0.65 & 1.05 & 1.22 & 3.78 & on-shell $H$ & 0.49 & 0.83 & 0.73 & 0 & 0.66 \\
\hline $13 \mathrm{TeV}$ & 0.66 & 0.64 & 1.03 & 1.19 & 3.51 & & & & & & \\
\hline
\end{tabular}

Table 1: Values of the $C_{1}$ factor in units $10^{-2}$ for the production modes for $p p$ collisions at centre-of-mass energies relevant for the LHC (left) and for the most relevant decay modes of the Higgs boson (right)

Denoting as $\Sigma$ a generic cross section, the corrections induced by an anomalous trilinear coupling modify the $\mathrm{LO}^{1}$ prediction $\left(\Sigma_{\mathrm{LO}}\right)$ according to

$$
\Sigma_{\mathrm{NLO}}=Z_{H} \Sigma_{\mathrm{LO}}\left(1+\kappa_{\lambda} C_{1}\right)
$$

where the coefficient $C_{1}$ depends on the process and the kinematical observable considered, while $Z_{H}$ is universal. $Z_{H}$ originates from the wave function renormalisation constant of the external Higgs field. In order to extend the range of convergence of the perturbative expansion to large values of $\kappa_{\lambda}$, the one-loop contribution in $Z_{H}$ has been resummed:

$$
Z_{H}=\frac{1}{1-\kappa_{\lambda}^{2} \delta Z_{H}}, \quad \delta Z_{H}=-\frac{9}{16} \frac{G_{\mu} m_{H}^{2}}{\sqrt{2} \pi^{2}}\left(\frac{2 \pi}{3 \sqrt{3}}-1\right) .
$$

In doing so, terms of $\mathscr{O}\left(\left(\kappa_{\lambda}^{2} \alpha\right)^{n}\right)$ which are expected to be the dominant higher-order corrections at large $\kappa_{\lambda}$ are correctly accounted for, however as a consequence we need to impose $\kappa_{\lambda}^{2} \delta Z_{H} \lesssim 1$, i.e., $\left|\kappa_{\lambda}\right| \lesssim 25$.

For each observable, the $C_{1}$ coefficient is identified as the contribution linearly proportional to $\lambda_{3}^{\mathrm{SM}}$ in the NLO EW corrections and normalised to the LO result as evaluated in the SM:

$$
C_{1}=\frac{\mathbb{Z} 2 \Re\left(\mathscr{M}^{0 *} \mathscr{M}_{\lambda_{3}^{\mathrm{sM}}}^{1}\right)}{\mathbb{L}\left|\mathscr{M}^{0}\right|^{2}}
$$

where $\mathscr{M}$ denotes a generic amplitude for single Higgs production or a Higgs decay width and the symbol $\mathbb{Z}$ implicates integration over phase space, convolution with parton distribution functions, sum/average of helicities and colour states. We refer to [1] for details on the computation of the $C_{1}$ coefficients of the various processes. The results are shown in table 1, while in Fig. 1 we plot the corrections to the relevant production cross sections $\delta \sigma_{\lambda_{3}}$ and decay branching ratios $\delta \mathrm{BR}_{\lambda_{3}}$ as functions of $\kappa_{\lambda}$.

\section{Results}

In this section we describe the method and the results of a simplified fit we have performed in order to estimate the limits that can be set on $\kappa_{\lambda}$ with our approach. Our analysis is based on the experimental results presented in Tab. 8 of Ref. [4]. We also estimate the expected limits that could be obtained at LHC Run-II at $300 \mathrm{fb}^{-1}$ and $3000 \mathrm{fb}^{-1}$ of luminosity.

\footnotetext{
${ }^{1}$ Here and in the following the LO contribution is understood as including QCD corrections so that the labels LO and NLO refer to EW corrections.
} 


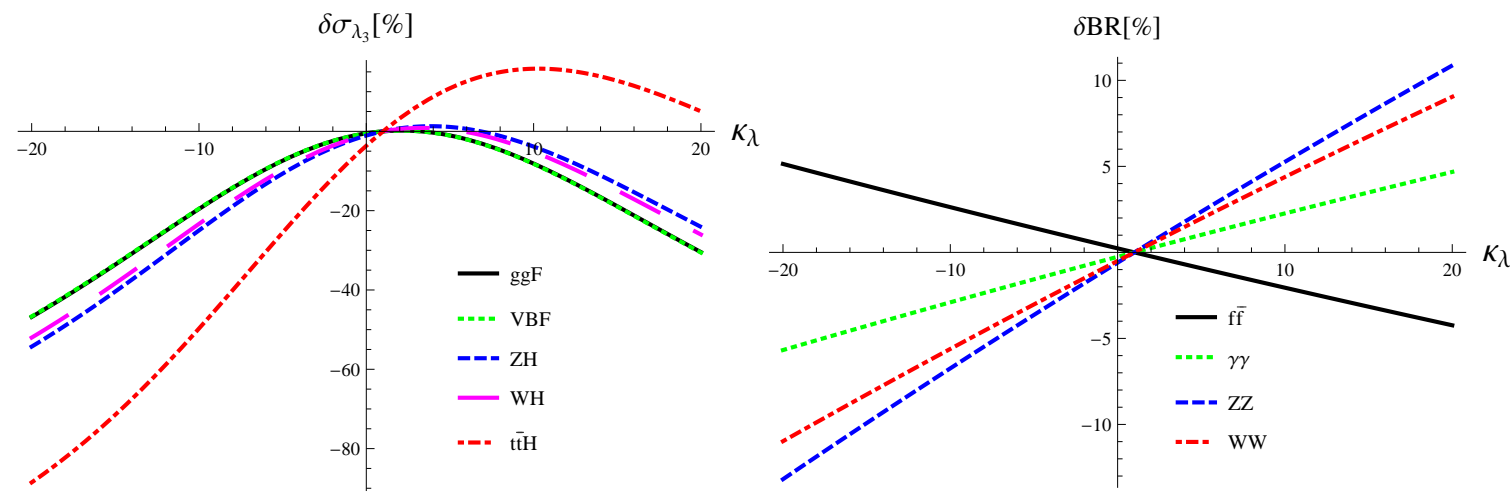

Figure 1: Left: Dependence of $\delta \sigma_{\lambda_{3}}$ (left) and $\delta \mathrm{BR}_{\lambda_{3}}$ (right) for the relevant production and decay processes.

In the following we denote the measured signal strengths as $\bar{\mu}_{i}^{f}$. Given a collection of $\bar{\mu}_{i}^{f}$ measurements $\left\{\bar{\mu}_{i}^{f}\right\}$, we define as best value of $\kappa_{\lambda}$ the one that minimises the $\chi^{2}\left(\kappa_{\lambda}\right)$ function defined as

$$
\chi^{2}\left(\kappa_{\lambda}\right) \equiv \sum_{\bar{\mu}_{i}^{f} \in\left\{\bar{\mu}_{i}^{f}\right\}} \frac{\left(\mu_{i}^{f}\left(\kappa_{\lambda}\right)-\bar{\mu}_{i}^{f}\right)^{2}}{\left(\Delta_{i}^{f}\left(\kappa_{\lambda}\right)\right)^{2}},
$$

where $\mu_{i}^{f}\left(\kappa_{\lambda}\right)$ are computed from the modified cross sections and branching ratios, and $\Delta_{i}^{f}\left(\kappa_{\lambda}\right)$ is the total uncertainty of $\mu_{i}^{f}$.
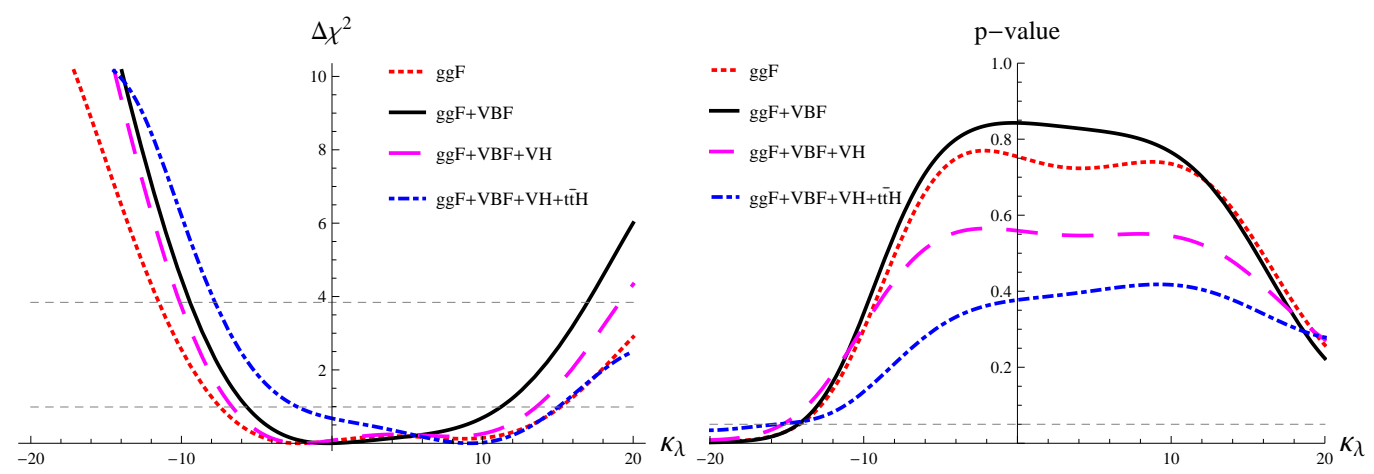

Figure 2: Left: $\chi^{2}$ for the different sets of observables. The two horizontal lines represent $\Delta \chi^{2}=1$ and $\Delta \chi^{2}=3.84$. Right: corresponding $p$-value. The horizontal line is $p=0.05$.

As shown in Fig. 2, we identify the $1 \sigma$ and $2 \sigma$ intervals assuming a $\chi^{2}$ distribution. Following this procedure and using the gluon-gluon-fusion and VBF data from Tab. 8 of Ref. [4] we obtain

$$
\kappa_{\lambda}^{\text {best }}=-0.24, \quad \kappa_{\lambda}^{1 \sigma}=[-5.6,11.2], \quad \kappa_{\lambda}^{2 \sigma}=[-9.4,17.0],
$$

where the $\kappa_{\lambda}^{\text {best }}$ is the best value and $\kappa_{\lambda}^{1 \sigma}, \kappa_{\lambda}^{2 \sigma}$ are respectively the $1 \sigma$ and $2 \sigma$ intervals.

In order to ascertain the goodness of our fit, we computed the $p$-value as a function of $\kappa_{\lambda}$ :

$$
\mathrm{p}-\operatorname{value}\left(\kappa_{\lambda}\right)=1-F_{\chi_{(n)}^{2}}\left(\chi^{2}\left(\kappa_{\lambda}\right)\right)
$$




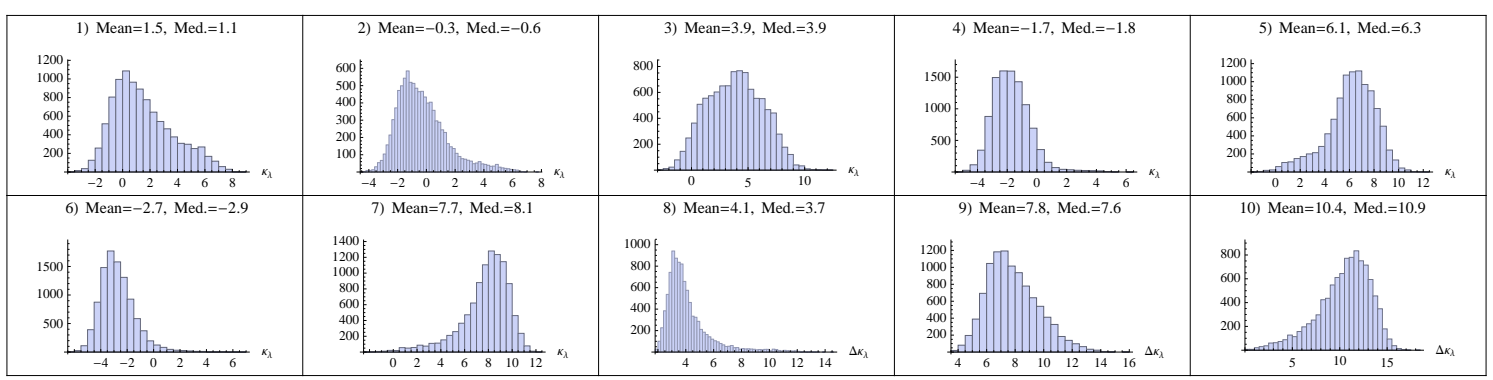

Figure 3: Histograms for "CMS-HL-II" $\left(3000 \mathrm{fb}^{-1}\right)$. The distributions represented are, from left to right and from top to bottom: 1) best values, 2) $1 \sigma$ region lower limit, 3) $1 \sigma$ region upper limit, 4) $2 \sigma$ region lower limit, 5) $2 \sigma$ region upper limit, 6) $p>0.05$ region lower limit, 7) $p>0.05$ region upper limit, 8) $1 \sigma$ region width, 9) $2 \sigma$ region width, 10) $p>0.05$ region width.

where $F_{\chi_{(n)}^{2}}\left(\chi^{2}\left(\kappa_{\lambda}\right)\right)$ is the cumulative distribution function for a $\chi^{2}$ distribution with $n$ degrees of freedom, computed at $\chi^{2}\left(\kappa_{\lambda}\right)$. In the right-hand side of Fig. 2 we report the $p$-value $\left(\kappa_{\lambda}\right)$ corresponding to different data sets. Requiring that $p>0.05$, we are able to exclude, at more than $2 \sigma$, that a model with an anomalous coupling $\kappa_{\lambda}<-14.3$ can explain the data.

We repeat the same procedure for ATLAS and CMS at $300 \mathrm{fb}^{-1}$ and $3000 \mathrm{fb}^{-1}$, using the uncertainties reported in Tab. 1 of [10] and, as a first step, assuming that the central value of the measurements in every channel coincides with the predictions of the SM. Within this approach, best values are by definition: $\kappa_{\lambda}^{\text {best }}=1$. For the $1 \sigma$ and $2 \sigma$ intervals, and for the region where the $p$-value is larger than 0.05 , we find that the "CMS-HL-II" $\left(3000 \mathrm{fb}^{-1}\right)$ case gives

$$
\kappa_{\lambda}^{1 \sigma}=[-0.7,4.2], \quad \kappa_{\lambda}^{2 \sigma}=[-2.0,6.8], \quad \kappa_{\lambda}^{p>0.05}=[-4.1,9.8] .
$$

This simplified approach provides a first (rough) idea of the typical intervals that can be expected. A more reliable approach consists of considering all the possible central values that could be measured. To this aim, we produce a collection of pseudo-measurements $\left\{\bar{\mu}_{i}^{f}\right\}$, where each $\bar{\mu}_{i}^{f}$ is randomly generated with a gaussian distribution around the SM with a standard deviation equal to the experimental uncertainty cited in Tab. 1 of [10]. For each pseudo-experiment we perform a fit and we determine $\kappa_{\lambda}^{\text {best }}$ and the $\kappa_{\lambda}^{1 \sigma}, \kappa_{\lambda}^{2 \sigma}$ and $\kappa_{\lambda}^{p>0.05}$ intervals. In Figs. 3 we report the results out of a collection of $n=10000$ pseudo-experiment. Frequency histograms together with corresponding mean and median values are provided for $\kappa_{\lambda}^{\text {best }}$ and all the extremes and widths of the $\kappa_{\lambda}^{1 \sigma}, \kappa_{\lambda}^{2 \sigma}$ and $\kappa_{\lambda}^{p>0.05}$ intervals. From these plots it is clear that most likely the limits written in Eq. (3.4) are pessimistic, and the LHC should be able to put even stronger bounds.

\section{Conclusions}

In this work we have put forward an alternative method, which relies on the effects that loops featuring an anomalous trilinear coupling would imprint on single Higgs production channels at the LHC. The bounds obtained are found to be competitive with the current ones obtained from Higgs pair production.

In all cases, the determination of the Higgs self coupling via loop effects is competitive with the direct determination and will provide complementary information. 


\section{Acknowledgements}

I would like to thank my collaborators, Giuseppe Degrassi, Fabio Maltoni and Davide Pagani for their permission to reproduce part of our joint work essentially verbatim in this proceedings. This work was supported by the United States Department of Energy under Grant Contracts desc0012704.

\section{References}

[1] G. Degrassi, P. P. Giardino, F. Maltoni and D. Pagani, Probing the Higgs self coupling via single Higgs production at the LHC, arXiv:1607.04251 [hep-ph].

[2] V. Khachatryan et al. [CMS Collaboration], Precise determination of the mass of the Higgs boson and tests of compatibility of its couplings with the standard model predictions using proton collisions at 7 and $8 \mathrm{TeV}$, Eur. Phys. J. C 75 (2015) no.5, 212 doi:10.1140/epjc/s10052-015-3351-7 [arXiv:1412.8662 [hep-ex]].

[3] G. Aad et al. [ATLAS Collaboration], Measurements of the Higgs boson production and decay rates and coupling strengths using pp collision data at $\sqrt{s}=7$ and $8 \mathrm{TeV}$ in the ATLAS experiment, Eur. Phys. J. C 76 (2016) no.1, 6 doi:10.1140/epjc/s10052-015-3769-y [arXiv:1507.04548 [hep-ex]].

[4] G. Aad et al. [ATLAS and CMS Collaborations], Measurements of the Higgs boson production and decay rates and constraints on its couplings from a combined ATLAS and CMS analysis of the LHC pp collision data at $\sqrt{s}=7$ and $8 \mathrm{TeV}$, JHEP 1608 (2016) 045 doi:10.1007/JHEP08(2016)045 [arXiv:1606.02266 [hep-ex]].

[5] A. David et al. [LHC Higgs Cross Section Working Group Collaboration], LHC HXSWG interim recommendations to explore the coupling structure of a Higgs-like particle, arXiv:1209.0040 [hep-ph].

[6] S. Heinemeyer et al. [LHC Higgs Cross Section Working Group Collaboration], Handbook of LHC Higgs Cross Sections: 3. Higgs Properties, doi:10.5170/CERN-2013-004 arXiv:1307.1347 [hep-ph].

[7] C. Anastasiou, C. Duhr, F. Dulat, E. Furlan, T. Gehrmann, F. Herzog, A. Lazopoulos and B. Mistlberger, High precision determination of the gluon fusion Higgs boson cross-section at the LHC, JHEP 1605 (2016) 058 doi:10.1007/JHEP05(2016)058 [arXiv:1602.00695 [hep-ph]].

[8] S. Borowka, N. Greiner, G. Heinrich, S. P. Jones, M. Kerner, J. Schlenk, U. Schubert and T. Zirke, Higgs Boson Pair Production in Gluon Fusion at Next-to-Leading Order with Full Top-Quark Mass Dependence, Phys. Rev. Lett. 117 (2016) no.1, 012001 Erratum: [Phys. Rev. Lett. 117 (2016) no.7, 079901] doi:10.1103/PhysRevLett.117.079901, 10.1103/PhysRevLett.117.012001 [arXiv:1604.06447 [hep-ph]].

[9] [CMS Collaboration], Projected Performance of an Upgraded CMS Detector at the LHC and HL-LHC: Contribution to the Snowmass Process, arXiv:1307.7135 [hep-ex].

[10] M. E. Peskin, Estimation of LHC and ILC Capabilities for Precision Higgs Boson Coupling Measurements, arXiv:1312.4974 [hep-ph]. 